\title{
STEATOHEPATITIS IN PATIENTS WITH INFLAMMATORY BOWEL DISEASES IN KARELIA
}

\author{
Olga P. Dudanova, Nadezhda A. Larina, Lidija V. Prokapovich, Anastasia A. Larina A. \\ Petrozavodsk State University, Petrozavodsk, Russia \\ O.P. Dudanova \\ 33 Lenin Avenue, Petrozavodsk, Russia 185910 \\ odudanova@gmail.com
}

\begin{abstract}
Background. Inflammatory bowel diseases (IBD) may be accompanied by the development of steatohepatitis $(\mathrm{SH})$, but its frequency and the dependence of liver damage on the form and severity of IBD can vary considerably according to observations of different researchers. The aim of the study was to evaluate the incidence and severity of $\mathrm{SH}$ in patients with ulcerative colitis (UC) and Crohn's disease (CD) in the Republic of Karelia.

Materials and methods. Sixty six patients with IBD (48 with UC and 18 with CD) were examined before administration of an anti-inflammatory therapy in order to exclude possible druginduced liver damage. There were 35 males $(53.0 \%)$ and 31 females (47.0\%), the mean age was 45.3 \pm 5.2 . IBD was diagnosed on the basis of clinical, laboratory and instrumental data (colonoscopy, upper gastrointestinal endoscopy with biopsies of tissue/lesions, computed tomography enterography, hydromagnetic resonance imaging of intestine, barium double-contrast enema radiographic studies). SH was diagnosed relying on laboratory tests such as ALT / AST (alanine/aspartate amino transferase), bilirubin, alkaline phosphatase and others, ultrasonography data and histological studies of liver biopsies or autopsies, and after the exclusion of hepatitis of viral and autoimmune origin.

Results. SH was revealed in $19(28.8 \%)$ patients with IBD: in $10(55.6 \%)$ CD patients and in $9(18.8 \%)$ UC patients. SH in IBD was characterized by mild (in $90.0 \%$ of patients) and moderate (in 10.0\%) inflammatory activity. Direct relationship between the development of SH and the activity of IBD $(r=0.60, p<0.05)$ or extent of IBD $(r=0.69, p<0.5)$ was observed. SH in CD patients was affected mainly by malabsorption, malnutrition and deterioration of enterohepatic circulation of bile acids, while SH in UC patients was affected predominantly by alteration in the intestinal microflora. Steatohepatitis activity decreased significantly in 4-8 weeks as a result of antiinflammatory treatment of bowel diseases without special hepatoprotective therapy.

Conclusion. Steatohepatitis, which aroused in $30.5 \%$ of patients with inflammatory bowel diseases, was characterized by mild activity and related to the extent of intestinal damage and inflammatory activity.
\end{abstract}

Keywords: inflammatory bowel diseases, Crohn's disease, ulcerative colitis, steatohepatitis. 


\section{Background}

Inflammatory bowel diseases (IBD), which include ulcerative colitis (UC) and Crohn's disease (CD), are relatively widely distributed among people over the world and Russian Federation. They are characterized by heavy course, frequent development of extraintestinal complications, and involvement of different organs and systems in pathological process including liver injury (Loftus, 1997; Danese, 2005; Navaneethan, 2010). Steatosis and steatohepatitis (SH) are considered the most common liver diseases, that appear in $15-88 \%$ of patients suffering from IBD, according to literature resources, and have favorable course (Dutt, 1983; Aitola, 1994; Broome, 1990; Bargiggia, 2003; Adamopoulos, 2006; Gaitini, 2011).

Pathogenesis of SH development in UC and CD has both common and distinctive features. Liver and intestine have tight anatomical and physiological connectives. First of all, it is necessary to mention the entero-hepatic axis. Bile acids that are synthesized by the liver are reabsorbed in the distal ileum and $95 \%$ of their quantity returns to the liver. The entero-hepatic bile acids circulation is disturbed in CD: the uptake of bile acids by enterocytes is decreased, endogen synthesis of toxic derivatives by hepatocytes is greatly increased, whereas bile acids efflux is decreased (Nolan, 2013). The bile acids are toxic detergents that can injure the hepatocytes and cause macrovesicular steatosis. Besides $\mathrm{CD}, \mathrm{SH}$ can accompany malabsorption, malnutrition and protein loss.

The liver receives $75 \%$ of blood flow from the intestine through the portal vein, and various proinflammatory cytokines, products of microbial vital activity, components of their surface structures, intestinal toxins therefore directly get to the liver (Vierling, 2003; Son, 2010; Szabo, 2010; Bawa, 2013). Kuppffer's cells are able to capture them and become activated, starting to release interleukins, free radicals and other biologically active molecules, which induce liver injuring and inflammation. SH can progress through different courses, but the majority of researches describe minimal and mild necro-inflammation and steatosis in the liver and detect benign clinical course of SH (Uko, 2012).

It is noteworthy that the researchers' opinion on correlation between the SH emergency and the activity or extent of colitis are contradictory (Broome, 1990; Riegler 1998; Uko, 2012). The prevalence of SH and its clinical peculiarities in patients with IBD in Karelia are largely unknown.

The purpose of this study was to determine the frequency of SH development in patients with IBD (ulcerative colitis and Crohn's disease) in Karelia, its clinical symptoms and characteristics of its course.

\section{Materials and methods}

66 patients with IBD (48 with UC and 18 with CD) who were treated in hospital clinics of Petrozavodsk from 2011 till 2013 were enrolled into the research. Males prevailed (27 persons, $56.3 \%$ ) among UC patients and females (10 persons, 55.5\%) - among CD patients. The mean age was $45.3 \pm 5.2$ years old in UC patients (range: 18-70) and $31.5 \pm 4.3$ in CD patients (range: 16-40); disease duration was $4.9 \pm 0.6$ (range: $0.5-7$ ) and 3.6 \pm 0.9 (range: $0.5-5$ ) years, respectively.

The design of the research included collection and processing of clinical, laboratory, instrumental and histological data. We evaluated the results of a blood count, erythrocyte sedimentation rate (ESR), levels of C-reactive protein (CRP) and fibrinogen, liver functional tests (alanine aminotransferase - ALT, aspartate aminotransferase - AST, alkaline phosphatase - APh, bilirubin, albumin, prothrombin) obtained by conventional laboratory methods. Dysbacteriosis analysis was performed using inoculation of feces on nutrient media.

Autoimmune hepatitis markers (antinuclear, antismoothmuscular, antimitohondrial, antineutrophil cytoplasmic antibodies - ANA, ASMA, AMA, ANCA) were determined by immuneenzyme analysis using "DRG Diagnostics" test-systems (Germany). Serological markers of HBVand $\mathrm{HCV}$-infections (HBsAg, AbHCV) were explored by immune-enzyme analysis using "VectorBest” test-systems (Novosibirsk, Russia). 
Colonoscopy and upper gastrointestinal endoscopy with biopsies of tissue/lesions were performed using a gastroscope and a colonoscope "Penthax", "HOYA" (Japan). Barium doublecontrast enema radiographic studies were performed in 50 patients. Computed tomographyenterography was performed in $5 \mathrm{CD}$ patients using the Aquilion 64 (Toshiba Medical Systems, Japan). Hydromagnetic resonance imaging of intestine was performed in 1 CD patient using MRT Magnum (USA).

Biopsies of gastrum, duodenum postbulbar region and intestinal mucosae were taken, and histological research was performed for all patients and samples. Histological research of intestinum sections, resected during surgery operation, was performed in 5 patients with CD. Histological research of liver tissue samples was performed for 6 patients: for 3 patients intravitally ( 2 with UC and 1 with CD) and for 3 patients postmortally ( 2 with CD and 1 with UC). Histological research of the intestinum was performed in 2 patients with $\mathrm{CD}$ and 1 patient with UC postmortally. Histological samples were stained with gematoxylin, eosin, and by Van-Gison method and were studied using the light microscope "LOMO Micmed-2" (St. Petersburg, Russia).

Abdominal ultasonography was carried out using an ultrasonic scanner "Philips En Visor" (USA) in order to estimate the liver size and echogenicity (graded as mild-to-moderate or severe, indicating a corresponding degree of hepatic steatosis).

Thirty patients (22 with UC, 8 - with CD) received monotherapy of 5-aminocalicilic acid (5ASA) (Salofalk, Sulphasalazine, Mesalasine), 13 patients $(7$ - with UC, 6 - with CD) were administered combined therapy of 5-ACA and corticosteroids (prednisolone), 5 patients (2 with UC and 3 with $\mathrm{CD}$ ) obtained combined therapy of 5-ACA, corticosteroids and cytostatics (asathioprine, methotrexate), and 5 patients with UC were treated locally by corticosteroids.

Statistical analysis was conducted using Statistics 6 software and Student's t-test, MannWhitney U-test and Spearman's rank correlation coefficient calculation. The level of statistical significance was defined as $\mathrm{p}<0.05$.

Exclusion criteria were antibiotic-associated colitis, ischemic colitis, irritable bowel syndrome and gluten entheropathy.

The design of research was approved by the ethic committee of medical faculty of Petrozavodsk State University.

\section{Results}

Steathohepatitis was revealed in $19(28.8 \%)$ IBD patients: in $10(55.6 \%)$ CD patients and in $9(18.8 \%)$ UC patients. Direct relationship between the development of SH and the activity or extent of IBD was observed. SH arose predominantly in patients with Crohn's disease exhibiting high (in $60.0 \%$ of patients) and moderate (in $40.0 \%$ of patients) inflammatory activity and did not arise in patients with mild inflammatory activity.

Besides the extent of inflammation, Crohn's disease also correlated with SH emergence. SH was more frequently observed in patients with the combined localization of the terminal ileum and some other parts of the intestine $(60.0 \%$ of patients) and less frequently in patients with isolated ileocecal localization of CD (40.0\% of patients).

The same pattern was observed in patients with UC. SH was predominantly detected in UC patients with high (in 2 persons, i.e. 22.2\%) and moderate (in 5 persons, i.e. $55.5 \%$ ) inflammatory activity and rarely - in UC patients with mild activity (in 2 persons, i.e. 22.2\%). UC extent influenced SH development: it was detected more frequently in cases of total and subtotal colitis (in 6 patients, or $66.7 \%$ ) and less frequently in cases of left-sided colitis (in 3 patients, 33.3\%).

Spearman's rank coefficient between emergency of SH and inflammatory activity of IBD was $0.60(p<0.05)$ and between emergency of SH and extent of IBD it was $0.69(p<0.05)$.

SH in IBD was characterized by mild and moderate inflammatory activity: for CD mild activity was detected in $90.07 \%$ of patients and moderate - in $10.0 \%$ of patients, and for UC - in $89.9 \%$ and $11.1 \%$ of patients, respectively. High activity SH was not observed among patients with $\mathrm{CD}$ and $\mathrm{UC}$ in the present study. 
Clinical symptoms of the SH were few and ambiguous. Only $10.0 \%$ of CD patients and $11.1 \%$ of UC patients complained of heaviness in the right hypohondrium. The symptoms of liver damage were not detected during physical examination of $\mathrm{CD}$ and UC patients.

At the same time, all IBD patients displayed altered functional liver tests: increased levels of transaminases or alkaline phosphatase and gamma-glutamiltranspeptidase (GGTP).

Abdominal ultrasonography revealed enlargement of liver size in $30.0 \%$ of CD patients and in $22.2 \%$ of UC patients, as well as enhanced echogenicity of the liver in $20.0 \%$ of CD patients and in $11.1 \%$ of UC patients.

The level of ALT in CD patients was higher than that in UC patients $-82.4 .6 \pm 17.5 \mathrm{U} / 1 \mathrm{vs}$. $52.8 \pm 10.2 \mathrm{U} / 1(\mathrm{p}<0.05)$, similar to the level of AST $-63.5 \pm 15.8 \mathrm{U} / 1 \mathrm{vs} .42 .7 \pm 9.4 \mathrm{U} / 1(\mathrm{p}<0.05)$, respectively (Table 1). Markers of intrahepatic cholestasis were higher in CD patients than those in UC patients: Aph $-238.5 \pm 12.3 \mathrm{U} / 1$ in CD vs. $189.4 \pm 8.6 \mathrm{U} / \mathrm{l}(\mathrm{p}<0.05)$ in UC, and GGTP $49.7 \pm 6.1$ vs. $39.5 \pm 4.1 \mathrm{U} / 1(\mathrm{p}<0.05)$, respectively.

There was a significant decrease in albumin level in CD patients $(23.0 \pm 3.1 \mathrm{~g} / \mathrm{l})$ compared to that found in UC patients $-33.1 \pm 1.5 \mathrm{~g} / \mathrm{l}$. This change was likely to be induced not only by the liver disease, but rather by a pronounced malabsorption syndrome in CD patients. Body mass index was $15.2 \pm 0.28 \mathrm{~kg} / \mathrm{m}^{2}$ in $\mathrm{CD}$ patients and $22.0 \pm 2.3 \mathrm{~kg} / \mathrm{m}^{2}(\mathrm{p}<0.05)$ in UC patients. Prothrombin level did not decrease either in CD patients $-78.2 \%$ or UC patients $-75.3 \%$, confirming that protein-synthetic liver function was not disturbed in both diseases.

Table 1. The results of functional liver tests in patients with inflammatory bowel diseases, $\mathrm{M} \pm \mathrm{m}$

\begin{tabular}{ccc}
\hline & $\mathrm{CD}, \mathrm{n}=10$ & UC, $\mathrm{n}=9$ \\
\hline $\mathrm{ALT}, \mathrm{U} / \mathrm{I}$ & $82.4 \pm 17.5$ & $52.8 \pm 10.2^{*}$ \\
\hline $\mathrm{AST}, \mathrm{U} / \mathrm{I}$ & $63.5 \pm 15.8$ & $42.7 \pm 9.4$ \\
\hline Aph, $\mathrm{U} / \mathrm{I}$ & $330.8 \pm 12.3$ & $189,4 \pm 18.6^{*}$ \\
\hline GGTP, $/ \mathrm{l}$ & $47.2 \pm 8.4$ & $39.5 \pm 4.1^{*}$ \\
\hline Bilirubin, $\mathrm{mkmol} / \mathrm{I}$ & $31.9 \pm 9.6$ & $25.2 \pm 10.3$ \\
\hline Albumin, $\mathrm{g} / \mathrm{l}$ & $23.0 \pm 3.1$ & $33.1 \pm 1.5^{*}$ \\
\hline Protrombin, \% & $78.2 \pm 8.1$ & $75.3 \pm 5.6$ \\
\hline * p $<0.05$ & &
\end{tabular}

Histological pattern of SH was characterized by macrovesicular or microvesicular steatosis of hepatocytes, and mild inflammatory infiltration of portal tracts (Figure 1).

Intestinal microbiota was characterized by decrease in numbers of Bifidobacteria and Lactobacteria and increase in opportunistic bacteria for both forms of IBD (Figure 2). At the same time the Bifidobacteria deficit was more evident in UC, than in CD patients, and the spectrum of opportunistic bacteria was more variable in UC, than in CD patients. 


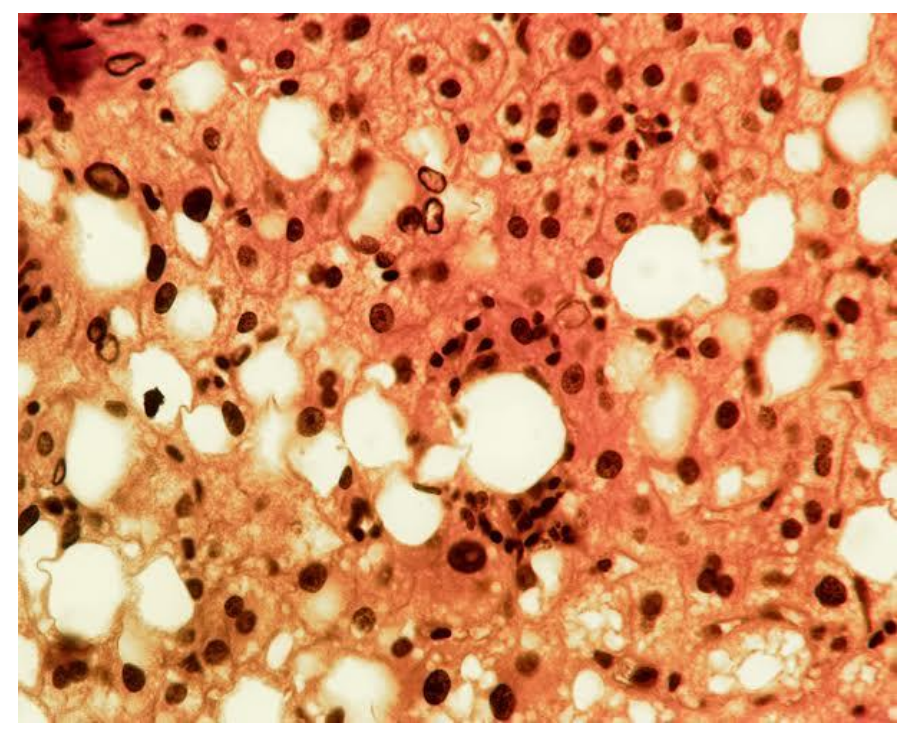

Figure 1. Macro- and microvesicular steatosis of hepatocytes, mild inflammatory infiltration in the vicinity of steatotic hepatocytes (stained with gematoxilin and eosin, magn. $x$ 200).

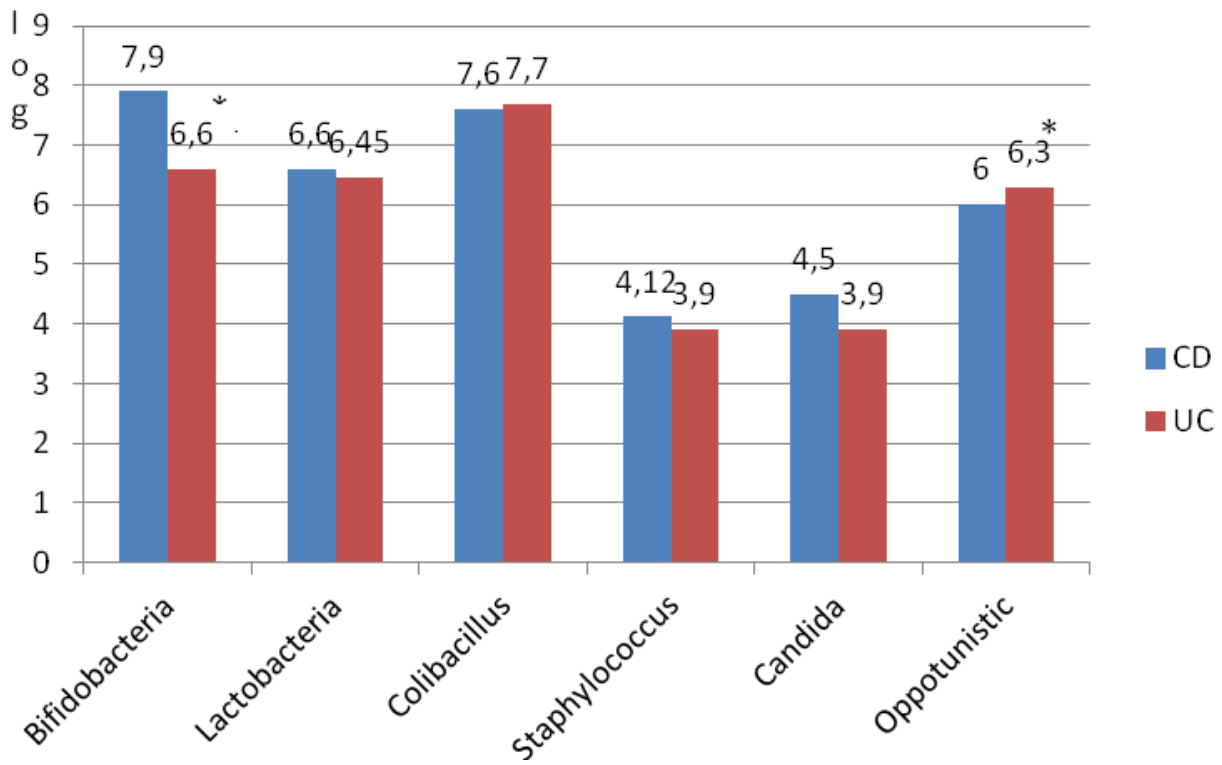

Figure 2. Quantitative composition of intestinal bacteria in CD and UC patients with steatohepatitis.

Citrobacter (50.0\%) and Enterobacter (25.0\%) prevailed in CD, Clebsiella was revealed in $12.5 \%$ and Proteus - in $6.0 \%$ of patients (Figure 3). More variable spectrum of opportunistic bacteria was revealed in UC patients: Proteus (24.2\%), Pseudomonas (24.2\%), Clebsiella (21.2\%), Enterobacter (24.2\%), and Citrobacter (6.0\%). It is commonly accepted that products of bacterial vital activity along with inflammatory cytokines damage the liver. Given the fact that the frequency of steatohepatitis in UC patients was significantly lower than that in CD patients, we can conclude that the disbalance of microbiota plays much smaller role in the pathogenesis of steatohepatitis than intestinal inflammation, malabsorbtion and impaired enterohepatic circulation of bile acids. 


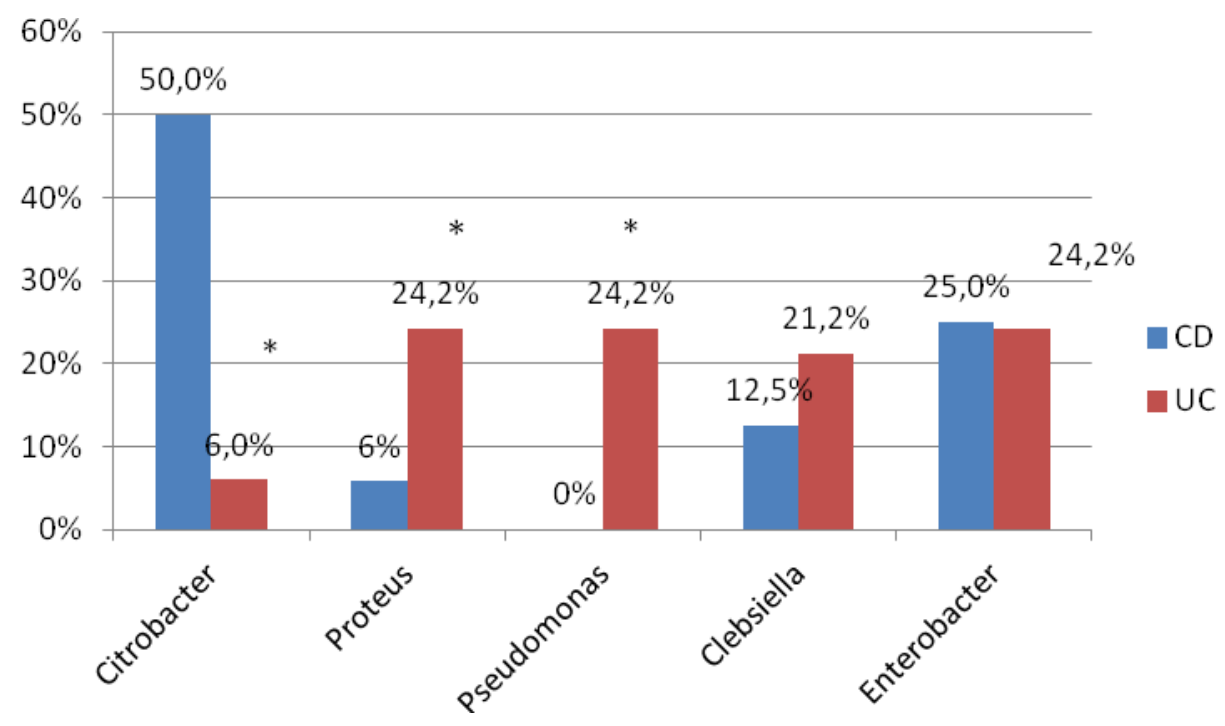

Figure 3. Composition of opportunistic bacteria in CD and UC patients with steatohepatitis.

Anti-inflammatory therapy led to the reduction of abdominal pain and stool frequency, improved stool consistency and decreased the levels of inflammatory markers in CD patients within 10 days. The counts of leucocytes, erythrocyte sedimentation rate (ESR), fibrinogen and Creactive protein (CRP) levels significantly reduced after 4 weeks (Table 2). At the same time, the values of functional liver tests (ALT, AST, APh) normalized due to elimination of inflammation in intestine; they decreased significantly within 4 weeks after the beginning of treatment and reached normal levels within 8 weeks.

Table 2. Results of anti-inflammatory therapy of IBD patients with steatohepatitis, $M \pm m$

\begin{tabular}{cccccc}
\hline & \multicolumn{3}{c}{$\mathrm{CD}, \mathrm{n}=10$} & \multicolumn{2}{c}{ UC, $\mathrm{n}=9$} \\
\cline { 2 - 6 } & $\begin{array}{c}\text { Before } \\
\text { treatment }\end{array}$ & In 4 weeks & In 8 weeks & $\begin{array}{c}\text { Before } \\
\text { treatment }\end{array}$ & In 4 weeks \\
\hline $\begin{array}{c}\text { Leucocytes, } \\
\mathrm{n} \times 10^{9} / \mathrm{l}\end{array}$ & $12.3 \pm 2.6$ & $8.1 \pm 1.5^{*}$ & $5.7 \pm 1.8$ & $8.2 \pm 2.3$ & $4.8 \pm 0.9^{*}$ \\
\hline $\mathrm{ESR}, \mathrm{mm} / \mathrm{h}$ & $31.3 \pm 4.6$ & $22.9 \pm 3.8^{*}$ & $13.2 \pm 2.1^{*}$ & $28.7 \pm 3.1$ & $15.6 \pm 2.5^{*}$ \\
\hline Fibrinogen, $\mathrm{g} / \mathrm{l}$ & $6.2 \pm 1.5$ & $5.6 \pm 0.9^{*}$ & $3.9 \pm 0.5^{*}$ & $5.7 \pm 1.9$ & $4.0 \pm 0.5$ \\
\hline $\mathrm{CRP}, \mathrm{mg} / \mathrm{l}$ & $21.0 \pm 9.0$ & $8.5 \pm 3.1^{*}$ & $5.6 \pm 2.2^{*}$ & $14.8 \pm 7.3$ & $3.5 \pm 0.3^{*}$ \\
\hline $\mathrm{ALT}, \mathrm{U} / \mathrm{l}$ & $82.4 \pm 17.5$ & $56.4 \pm 18.2^{*}$ & $35.6 \pm 5.5^{*}$ & $71.3 \pm 13.8$ & $35.6 \pm 6.8^{*}$ \\
\hline $\mathrm{AST}, \mathrm{U} / \mathrm{l}$ & $63.5 \pm 15.8$ & $41.4 \pm 13.4^{*}$ & $28.3 \pm 2.6^{*}$ & $67.5 \pm 15.4$ & $19.8 \pm 4.7$ \\
\hline $\mathrm{APh}, \mathrm{U} / \mathrm{l}$ & $330.8 \pm 22.3$ & $278.0 \pm 19.1^{*}$ & $235.8 \pm 20.4$ & $199.8 \pm 23.5$ & $133.2 \pm 18.1^{*}$ \\
\hline$* \mathrm{p}<0.05$ & & & & &
\end{tabular}

Clinical and laboratory dynamics in UC patients was more prominent, than in CD patients. Abdominal pain, stool frequency decreased by the end of the first week of treatment. Inflammatory tests (leucocytes, ESR, CRP, fibrinogen) significantly decreased in 4 weeks (Table 2). At the same time, the levels of functional liver tests (ALT, AST) reached the norm. Although $\mathrm{APh}$ did not exceed the normal level in UC patients initially as opposed to CD patients, its activity significantly decreased in 4 weeks. 


\section{Conclusion}

Steatohepatitis aroused in $28.8 \%$ of patients with inflammatory bowel diseases: in $55.6 \%$ of patients with Crohn disease and in $18.8 \%$ of patients with ulcerative colitis.

The risk of steatohepatitis emergence in both diseases mainly depended on the extent of intestinal damage and inflammatory activity.

In addition, steatohepatitis in $\mathrm{CD}$ patients depended on malnutrition, deterioration of hepatobiliary circulation of bile acids, and in UC patients - on alterations in the intestinal microbiota.

Steatohepatitis in inflammatory bowel diseases was characterized by mild activity in $90.0 \%$ of patients.

Steatohepatitis activity decreased significantly in 4 weeks in ulcerative colitis and in 8 weeks in Crohn's disease due to anti-inflammatory treatment of bowel diseases without using of special hepatoprotective therapy.

\section{References}

1. Adamopoulos AB. Inflammatory Bowel Disease and the Liver. Annals of Gastroenterology 2006, 19(2):146-151.

2. Aitola P, Karvonen AL, Matikainen M. Prevalence of hepatobiliary dysfunction in patients with ulcerative colitis. Ann Chir Gynecol 1994, 83:275-278.

3. Bargiggia S, Maconi G, Elli M, Molteni P, Ardizzone S, Parente F, Todaro I, Greco S, Manzionna G, Bianchi Porro G. Sonographic prevalence of liver steatosis and biliary tract stones in patients with inflammatory bowel disease: study of 511 subjects at a single center. $J$ Clin Gastroenterol 2003, 36(5):417-420.

4. Bawa M, Saraswa VA. Gut-Liver Axis: role of inflammasomes. J Clinical and Exper Hepatology 2013, 3(2):141-149.

5. Broome U, Glaumann H, Hultcrantz R. Liver histology and follow up of 68 patients with ulcerative colitis and normal liver function tests. Gut 1990, 31(4):468-472.

6. Danese S, Semeraro S, Papa A, Roberto I, Scaldaferri F, Fedeli G, Gasbarrini G, Gasbarrini A. Extraintestinal manifestations in inflammatory bowel disease. World J Gastroenterol 2005, 11(46):7227-7236.

7. Dutt MK, Davies DR, Grace RH, Thompson RP. Sampling variability of liver biopsy in inflammatory bowel disease. Arch Pathol Lab Med 1983, 107(9):451-452.

8. Gaitini D, Kreitenberg AJ, Fischer D, Maza I, Chowers Y. Color-coded duplex sonography compared to multidetector computed tomography for the diagnosis of Crohn disease relapse and complications. J Ultrasound Med 2011, 30:1691-1699.

9. Loftus EV Jr, Sandborn J, Lindor KD, Larusso NF. Interactions Between Chronic Liver Disease and Inflammatory Bowel Disease. Inflammatory Bowel Diseases 1997, 3(4):288-302.

10. Navaneethan U, Shen B. Hepatopancreatobiliary manifestations and complications associated with inflammatory bowel disease. Inflamm Bowel Dis 2010, 16(9): 1598-1619.

11. Nolan JD, Johnston IM, Walters JR. Altered enterohepatic circulation of bile acids in Crohn's disease and their clinical significance: a new perspective. Expert Rev Gastroenterol Hepatol 2013, 7(1):49-56.

12. Riegler G, D'Inc’a R, Sturniolo GC, Corrao G, Del Vecchio Blanco C, Di Leo V, Carratù R, Ingrosso M, Pelli MA, Morini S, Valpiani D, Cantarini D, Usai P, Papi C, Caprilli R. Hepatobiliary alterations in patients with inflammatory bowel disease: a multicenter study. Scandinavian J of Gastroenterology 1998, 33(1):93-98.

13. Son G, Kremer M, Hines IN. Contribution of Gut Bacteria to Liver Pathobiology. Gastroenterology Research and Practice 2010, doi: 10.1155/2010/453563. 
14. Szabo G, Bala S, Petrasek J, Gattu A. Gut-liver axis and sensing microbes. Dig Dis 2010, 28: 737-744.

15. Uko V, Thangada S, Radhakrishnan K. Liver Disorders in Inflammatory Bowel Disease. Gastroenterology Research and Practice 2012, doi: 10.1155/2012/642923.

16. Vierling JM. Hepatobiliary complications of ulcerative colitis and Crohn's disease. In Hepatology (textbook). 4th edition. Edited by Zakim \& Boyer. Philadelphia: Saunders Elsevier; 2003:1238-1240. 